\title{
ON A PLANAR AREA-PRESERVING CURVATURE FLOW
}

\author{
XIAO-LI CHAO, XIAO-RAN LING, AND XIAO-LIU WANG \\ (Communicated by Lei $\mathrm{Ni}$ )
}

\begin{abstract}
A classical nonlocal curvature flow preserving the enclosed area is reinvestigated. The uniform upper bound and lower bound of curvature are established for the first time. As a result, a detailed proof is presented for the asymptotic behavior of the flow.
\end{abstract}

\section{Preliminaries}

As a variation of the popular curve shortening flow [6, 17, 2, 4, 18, 19, 3], the nonlocal curvature flow, arising in many application fields [17, such as phase transitions, image processing, etc., has received much attention in recent years. The relevant research is focused on so-called area-preserving flow [5, 13, perimeterpreserving flow 16, 14, and other analogues [8, 11, 12, 15. The relations between these nonlocal flows are nicely summarized by Lin and Tsai [10].

The purpose of this paper is to reinvestigate the area-preserving flow evolving under

$$
\begin{aligned}
& \frac{\partial X}{\partial t}=\left(k-\frac{2 \pi}{L(t)}\right) N, \\
& X(u, 0)=X_{0}(u),
\end{aligned}
$$

where $X(u, t)=(x(u, t), y(u, t)):[a, b] \times[0, T) \rightarrow \mathbb{R}^{2}(T>0)$ is a family of evolving curves with signed curvature $k$, inward pointing unit normal $N$, length $L(t)$ and smooth initial data $X_{0}$. This flow has been studied by Gage [5] in 1986. It preserves the enclosed area $A(t)$ (also denoted by $A_{0}$ ) and has the property as follows.

Main Theorem. A simple closed convex plane curve which evolves according to (11) remains so and converges to a circle with radius $\sqrt{A_{0} / \pi}$ in the $C^{\infty}$ metric.

In this paper, a detailed proof of the main theorem is presented, which is based on a time-independent uniform estimate for the curvature of evolving curves. Our paper is composed of three parts. First, some known results are collected, which are coming next. Subsequently, the upper bound and lower bound of the curvature are established in Section 2. Finally, the convergence result is proved in the last section.

Since each curve $X(u, t)$ is strictly convex, each point on it has a unique tangent and one can use the tangent angle $\theta \in[0,2 \pi]$ to parameterize it. Generally speaking,

Received by the editors August 24, 2011.

2010 Mathematics Subject Classification. Primary 53C44; Secondary 35B40, 35K59, 37B25.

Key words and phrases. Mean curvature flow, nonlocal flow, asymptotic behavior.

This work was supported by PRC Grants NSFC 11101078, 10971029 and 11171064 and the Natural Science Foundation of Jiangsu Province BK2011583. 
$\theta$ is a function of $u$ and $t$. In order to make $\theta$ independent of time $t$, one can attain that by adding a tangential component to the velocity vector $\partial X / \partial t$, which does not affect the geometric shape of the evolving curve (see, for example, [5, 4]). Then the evolution equation for curvature can be expressed in terms of $\theta$ and $t$,

$$
k_{t}=k^{2} k_{\theta \theta}+k^{3}-\frac{2 \pi k^{2}}{L}, \quad \theta \in[0,2 \pi], t>0 .
$$

We would like to note that if the curvature function $k(\theta)$ of a closed curve $X(\theta)$ satisfies $k(\theta)>0, \theta \in[0,2 \pi]$, then $X(\theta)$ can be expressed by

$$
X(\theta)=X(0)+\left(\int_{0}^{\theta} \frac{\cos \theta}{k(\theta)} d \theta, \int_{0}^{\theta} \frac{\sin \theta}{k(\theta)} d \theta\right), \theta \in[0,2 \pi] .
$$

For the flow under (11) with a simple closed convex initial curve $X_{0}$, except its global existence, preserving area and convexity, Gage [5] has shown that its length $L(t)$ is decreasing, that is, $L^{\prime}(t) \leq 0$ for any $t>0$, and its isoperimetric deficiency satisfies

$$
\frac{\pi^{2}}{A}\left(r_{\text {out }}-r_{\text {in }}\right)^{2} \leq \frac{L^{2}}{A}-4 \pi \leq C e^{-\frac{2 \pi}{A} t}, \forall t>0,
$$

where $r_{\text {in }}$ and $r_{\text {out }}$ denote respectively the radii of the largest inscribed circle and the smallest circumscribed circle of the curve $X(\theta, t)$, and $C$ depends only on initial curve $X_{0}$.

\section{Estimates}

Note that (2) implies that the flow (11) must converge to a circle in the Hausdorff metric. To show the convergence in the $C^{\infty}$ metric, it is equivalent to show that the curvature function $k(\theta, t)$ converges to a constant in the $C^{\infty}$ sense. For this goal, we will establish a time-independent uniform estimate for the curvature of evolving curves in this section, which is comprised of an upper bound and a positive lower bound.

Our method to establish the upper bound of the curvature function is based on the literature [1, 6, 14. Some lemmas are prepared.

Definition 1. For fixed $w \in(0, \pi]$, we define $k_{w}^{*}(t)=\sup \{b \mid k(\theta, t)>b$ on some interval of length $w\}$.

Lemma 1 (Gage-Hamilton [6]). For any $t>0$ and $w \in(0, \pi]$, we have

$$
k_{w}^{*}(t) r_{\text {in }}(t) \leq \frac{1}{1-K(w)\left(\frac{r_{\text {out }}}{r_{\text {in }}}-1\right)}, \text { where } K(w)=\frac{2 \cos \frac{w}{2}}{1-\cos \frac{w}{2}} .
$$

Lemma 2. For fixed $w \in(0, \pi]$ there exists a time $T_{0}$ depending on $w$ such that

$$
k_{w}^{*}(t) \leq \sqrt{\frac{2 \pi}{A(0)}} \text { for all } t \geq T_{0} .
$$

Proof. From [5] we already know that $r_{\text {out }}(t) / r_{i n}(t) \rightarrow 1$ and $r_{i n}(t) \rightarrow \sqrt{A(0) / \pi}$ as $t \rightarrow \infty$. Since for fixed $w \in(0, \pi]$ we have

$$
\lim _{t \rightarrow \infty} K(w)\left(\frac{r_{\text {out }}(t)}{r_{\text {in }}(t)}-1\right)=0,
$$

the result follows by Lemma 1 
Lemma 3. For any $T>0$, if $k(\theta, t) \leq M$ on $[0,2 \pi] \times[0, T]$, then $M \geq 2 \pi / L(0)$ and

$$
\int_{0}^{2 \pi} k_{\theta}^{2}(\theta, t) d \theta \leq \tilde{C} M^{2} \text { for all } t \in[0, T]
$$

where $\tilde{C}>0$ is a constant depending only on the initial data. It is independent of $T$ and $M$.

Proof. By

$$
2 \pi=\int_{X(., t)} k d s \leq k_{\max }(t) L(t) \leq M L(0)
$$

we have $M \geq 2 \pi / L(0)$. Also it has been shown in 5 that there exist positive constants $D, C$ depending only on the initial curve such that

$$
\int_{0}^{2 \pi} k_{\theta}^{2}(\theta, t) d t \leq 2 \pi M^{2}+D M+C=M^{2}\left(2 \pi+\frac{1}{M} D+\frac{1}{M^{2}} C\right), \forall t \in[0, T] .
$$

The proof is done since $1 / M \leq L(0) /(2 \pi)$.

By the above lemma, we can obtain the uniform upper bound of the curvature:

Theorem 1. We have $k(\theta, t) \leq B_{1}$ on $[0,2 \pi] \times[0, \infty)$, where $B_{1}$ is a constant independent of time.

Proof. Fix a number $w \in(0, \pi]$ so that $\sqrt{w \tilde{C}}<\frac{1}{2}$, where $\tilde{C}$ is the constant in Lemma 3. Thus, by Lemma 2 there exists a time $T_{0}>0$ such that $k_{w}^{*}(t) \leq$ $\sqrt{2 \pi / A(0)}$ for $t \geq T_{0}$. Now we divide the interval $[0, \infty)$ into $\left[0, T_{0}\right]$ and $\left[T_{0}, \infty\right]$. For each fixed time $s \in\left(T_{0}, \infty\right)$, the open set $U=\left\{\theta: k(\theta, s)>k_{w}^{*}(s)\right\}$ can be written uniquely as the union of a countable number of disjoint open intervals $I_{i}$, each of which has length less than or equal to $w$ due to the definition of $k_{w}^{*}$. There exists some interval $I=(a, b)$ such that $|b-a| \leq w, k(a, s)=k_{w}^{*}(s)$ and $\theta(s) \in(a, b)$, where $k(\theta(s), s)=k_{\max }(s)$. Then we have

$$
k_{\max }(s)=k(a, s)+\int_{a}^{\theta(s)} k_{\theta} d \theta \leq k_{w}^{*}(s)+\sqrt{w}\left(\int_{0}^{2 \pi} k_{\theta}^{2}(\theta, s) d \theta\right)^{1 / 2} .
$$

Note that (3) is valid for any time $s \in\left(T_{0}, \infty\right)$.

For time $T>T_{0}$, set $M=\max _{[0,2 \pi] \times[0, T]} k(\theta, t)=k_{\max }\left(t_{*}\right)$ for some time $t_{*} \in[0, T]$. We may assume that $t_{*}>T_{0}$ (otherwise $M \leq \max _{[0,2 \pi] \times\left[0, T_{0}\right]} k(\theta, t)$ and this is essentially what we want). Hence

$$
\begin{aligned}
M & =k_{\max }\left(t_{*}\right) \leq k_{w}^{*}\left(t_{*}\right)+\sqrt{w}\left(\int_{0}^{2 \pi} k_{\theta}^{2}\left(\theta, t_{*}\right) d \theta\right)^{1 / 2} \\
& \leq \sqrt{\frac{2 \pi}{A(0)}}+\sqrt{w \tilde{C}} M \leq \sqrt{\frac{2 \pi}{A(0)}}+\frac{M}{2}
\end{aligned}
$$

which gives a uniform upper bound of $M$. As $T>T_{0}$ is arbitrary, the proof is done. 
As a consequence of the upper bound obtained already for the curvature function, we can establish the lower bound as follows. The method is from Andrews [1]. We first have:

Lemma 4. Under the area-preserving flow (11) we have

$$
\sup _{S^{1} \times[0, t]}\left(k_{\theta}^{2}+k^{2}\right) \leq \max \left\{\sup _{S^{1} \times[0, t]} k^{2}, \sup _{S^{1} \times\{0\}}\left(k_{\theta}^{2}+k^{2}\right)\right\}
$$

for all $t \in[0, \infty)$.

Proof. The proof is analogous to Lemma I1.12 in Andrews [1]. Let $\Phi=\left(k_{\theta}\right)^{2}+k^{2}$ and let $t>0$ be fixed. Suppose at $\left(\theta_{0}, t_{0}\right) \in S^{1} \times[0, t]$ we have $\Phi\left(\theta_{0}, t_{0}\right)=$ $\sup _{S^{1} \times[0, t]}\left(k_{\theta}^{2}+k^{2}\right)$. Then we may assume $t_{0}>0$ (otherwise we are done). We claim that $k_{\theta}\left(\theta_{0}, t_{0}\right)=0$. If not, then at $\left(\theta_{0}, t_{0}\right)$ we will have the following properties:

$$
\left\{\begin{array}{l}
k_{\theta \theta}+k=0 \\
\Phi_{\theta \theta}=2 k_{\theta}\left(k_{\theta \theta \theta}+k_{\theta}\right) \leq 0 \\
\frac{\partial \Phi}{\partial t}=2 k_{\theta} k^{2}\left(k_{\theta \theta \theta}+k_{\theta}\right)-4 k k_{\theta}^{2} \frac{2 \pi}{L}-2 k^{3} \frac{2 \pi}{L} \geq 0
\end{array}\right.
$$

which gives a contradiction. Hence $k_{\theta}\left(\theta_{0}, t_{0}\right)=0$ and we conclude that

$$
\sup _{S^{1} \times[0, t]}\left(k_{\theta}^{2}+k^{2}\right)=k^{2}\left(\theta_{0}, t_{0}\right) \leq \sup _{S^{1} \times[0, t]} k^{2} .
$$

The proof is done.

Theorem 2. We have $k(\theta, t) \geq B_{2}$ on $[0,2 \pi] \times[0, \infty)$, where $B_{2}$ is a constant independent of time.

Proof. By (4) and the uniform upper bound of the curvature, there is a constant $C$ independent of time such that

$$
\left|k_{\theta}(\theta, t)\right| \leq C \text { for all }(\theta, t) \in S^{1} \times[0, \infty) .
$$

This implies that

$$
\left|\log \frac{k\left(\theta_{2}, t\right)}{k\left(\theta_{1}, t\right)}\right|=\left|\int_{\theta_{1}}^{\theta_{2}} \frac{k_{\theta}(\theta, t)}{k(\theta, t)} d \theta\right| \leq C L(t) \leq C L(0)
$$

for all $t>0$ and any $\theta_{1}, \theta_{2} \in S^{1}$. In particular we have the Harnack-type estimate:

$$
\frac{k_{\max }(t)}{k_{\min }(t)} \leq e^{C L(0)}
$$

Also by

$$
\frac{2 \pi}{k_{\max }(t)} \leq \int_{0}^{2 \pi} \frac{1}{k(\theta, t)} d \theta=L(t) \leq L(0)
$$

we have

$$
k_{\min }(t) \geq k_{\max }(t) e^{-C L(0)} \geq \frac{2 \pi}{L(0)} e^{-C L(0)} .
$$

The proof is done. 


\section{Convergence}

In this section, we show the convergence of the flow under (1) via the Lyapunov functional method and complete the proof of the main theorem. We first claim:

Lemma 5. Under the area-preserving flow (11) we have

$$
\frac{d L}{d t}(t) \rightarrow 0 \text { as } t \rightarrow \infty
$$

Proof. Assume not. Since $d L(t) / d t \leq 0$, there exist a constant $C_{0}>0$ and a sequence of time $\left\{t_{i}\right\}_{i=1}^{\infty} \nearrow \infty$ such that

$$
\frac{d L}{d t}\left(t_{i}\right) \leq-C_{0} \text { for all } i=1,2,3, \cdots .
$$

Also note that

$$
\left|\frac{d^{2} L}{d t^{2}}(t)\right|=\left|\frac{d^{2}}{d t^{2}} \int_{0}^{2 \pi} \frac{1}{k(\theta, t)} d \theta\right| \leq \int_{0}^{2 \pi}\left|\frac{k_{t t}}{k^{2}}-\frac{2 k_{t}^{2}}{k^{3}}\right| d \theta
$$

and by the regularity estimate for quasilinear uniformly parabolic equations, the curvature $k(\theta, t)$ has all of its space-time derivatives uniformly bounded on $S^{1} \times$ $[0, \infty)$ (see Remark 7 in Lin-Tsai 9 or any parabolic PDE books). Hence there exists a constant $\lambda>0$ such that $\left|d^{2} L(t) / d t^{2}\right| \leq \lambda$ for all $t \in[0, \infty)$. As the slope of $L^{\prime}(t)$ is uniformly bounded, one can find a number $\rho_{0}$ independent of $t_{i}$ such that

$$
\left|\frac{d L}{d t}(t)\right| \geq \frac{C_{0}}{2} \text { for all } t \in\left[t_{i}, t_{i}+\rho_{0}\right] .
$$

This will imply that

$$
L(\infty)-L(0)=\int_{0}^{\infty} \frac{d L}{d t}(t) d t=-\infty
$$

a contradiction.

Next we prove the final convergence:

Theorem 3. The following estimate holds:

$$
\lim _{t \rightarrow \infty}\left\|k(\theta, t)-\sqrt{\frac{\pi}{A(0)}}\right\|_{C^{k}\left(S^{1}\right)}=0
$$

for any $k \in \mathbb{N}$.

Proof. By the regularity estimate, it suffices to prove $C^{0}$ convergence. For any sequence of time $\left\{t_{i}\right\}_{i=1}^{\infty}$ going to infinity, by the Arzelà-Ascoli theorem, there is a subsequence (still denoted by $\left\{t_{i}\right\}_{i=1}^{\infty}$ ) such that $k\left(\theta, t_{i}\right) \rightarrow K(\theta)$ in $C^{\infty}\left(S^{1}\right)$ as $i \rightarrow \infty$. Moreover,

$$
\begin{aligned}
\frac{d L}{d t}\left(t_{i}\right) & =-\int_{0}^{2 \pi} k\left(\theta, t_{i}\right) d \theta+\frac{4 \pi^{2}}{L\left(t_{i}\right)}=-\int_{0}^{2 \pi} k\left(\theta, t_{i}\right) d \theta+\frac{4 \pi^{2}}{\int_{0}^{2 \pi} \frac{1}{k\left(\theta, t_{i}\right)} d \theta} \\
& \rightarrow-\int_{0}^{2 \pi} K(\theta) d \theta+\frac{4 \pi^{2}}{\int_{0}^{2 \pi} \frac{1}{K(\theta)} d \theta}=0 \text { as } i \rightarrow \infty
\end{aligned}
$$

Therefore

$$
\int_{0}^{2 \pi} K(\theta) d \theta \int_{0}^{2 \pi} \frac{1}{K(\theta)}=4 \pi^{2}
$$


By the Hölder inequality we must have $K(\theta)=$ const. and since $L(t) \rightarrow \sqrt{4 \pi A(0)}$ as $t \rightarrow \infty$, we obtain $K(\theta)=\sqrt{\pi / A(0)}$.

Finally, if there exists a sequence of time $\left\{t_{i}\right\}_{i=1}^{\infty}$ such that $k\left(\theta, t_{i}\right)$ does not converge to $\sqrt{\pi / A(0)}$, then one can find a further subsequence such that along it we have convergence to $\sqrt{\pi / A(0)}$. This gives a contradiction. As a result, we must have convergence to $\sqrt{\pi / A(0)}$ as $t \rightarrow \infty$.

\section{ACKNOWLEDGMENTS}

The authors would like to thank the anonymous referee, who spent a lot of effort helping improve the presentation of this paper. They also thank Prof. Kaiseng Chou for his constant encouragement and enthusiastic guidance during the preparation of this work.

\section{REFERENCES}

[1] B. Andrews, Evolving convex curves, Calc. Var. Partial Differential Equations 7 (1998) 315371. MR1660843 (99k:58038)

[2] S. Angenent, Parabolic equations for curves on surfaces. I. Curves with $p$-integrable curvature, Ann. of Math.(2) 132 (1990) 451-483; Parabolic equations for curves on surfaces II. Intersections, blow-up and generalized solutions, Ann. of Math. (2) 133 (1991) 171-215. MR.1078266 (91k:35102), MR:1087347 (92b:58039)

[3] K.S. Chou, X.L. Wang, The curve shortening problem under Robin boundary condition, NoDea Nonlinear Differential Equations and Applications 19 (2012) 177-194. MR2902186

[4] K.S. Chou, X.P. Zhu, The curve shortening problem. Chapman \& Hall/CRC, Boca Raton, FL, 2001. MR 1888641 (2003e:53088)

[5] M. Gage, On an area-preserving evolution equation for plane curves, Nonlinear problems in geometry (Mobile, Ala., 1985), 51-62, Contemp. Math., 51, Amer. Math. Soc., Providence, RI, 1986. MR848933 (87g:53003)

[6] M. Gage, R. Hamilton, The heat equation shrinking convex plane curves, J. Differential Geom. 23 (1986) 69-96. MR840401 (87m:53003)

[7] M.A. Grayson, The heat equation shrinks embedded plane curves to round points, J. Differential Geom. 26 (1987) 285-314. MR906392 (89b:53005)

[8] L.S. Jiang, S.L. Pan, On a non-local curve evolution problem in the plane, Comm. Anal. Geom. 16 (2008) 1-26. MR2411467 (2009e:53083)

[9] Y.C. Lin, D.H. Tsai, On a simple maximum principle technique applied to equations on the circle, J. Diff. Eq. 245 (2008) 377-391. MR2428003 (2010b:35252)

[10] Y.C. Lin, D.H. Tsai, Nonlocal flow of convex plane curves and isoperimetric inequalities, Arxiv: $1005.0438 \mathrm{v} 1,2010$.

[11] Y.C. Lin, D.H. Tsai, On a general linear nonlocal curvature flow of convex plane curves, Arxiv: $1012.0114 \mathrm{v} 1,2010$.

[12] Y.C. Lin, D.H. Tsai, Application of Andrews and Green-Osher inequalities to nonlocal flow of convex plane curves, preprint, 2012.

[13] L. Ma, L. Cheng, A non-local area preserving curve flow, Arxiv: 0907.1430v1, 2009.

[14] L. Ma, A.Q. Zhu, On a length preserving curve flow, Monatshefte für Mathematik 165 (2012) 57-78. MR2886123

[15] S.L. Pan, H. Zhang, On a curve expanding flow with a nonlocal term, Comm. Contemp. Math. 12 (2010) 815-829. MR2733199 (2012c:53102)

[16] S.L. Pan, J.N. Yang, On a non-local perimeter-preserving curve evolution problem for convex plane curves, Manuscripta Math. 127 (2008) 469-484. MR2457190 (2010h:53099)

[17] G. Sapiro, A. Tannenbaum, Area and length preserving geometric invariant scale-spaces, Pattern Analysis and Machine Intelligence, IEEE Transactions 17 (1995) 67-72. 
[18] X.L. Wang, The stability of $m$-fold circles in the curve shortening problem, Manuscripta Math. 134 (2011) 493-511. MR2765723(2012d:53221)

[19] X.L. Wang, W.F. Wo, On the stability of stationary line and grim reaper in planar curvature flow, Bulletin of the Australian Mathematical Society 83 (2011) 177-188. MR2784776 (2012c:35208)

Department of Mathematics, Southeast University, Nanjing, People's Republic of CHINA

Department of Mathematics, Southeast University, Nanjing, People's Republic of CHINA

Department of Mathematics, Southeast University, Nanjing, People's Republic of CHINA 